\title{
Pre-Day Scheduling of Charging Processes in Mobility-on-Demand Systems Considering Electricity Price and Vehicle Utilization Forecasts
}

\author{
Florian Dandl ${ }^{1,2}$, Fabian Fehn ${ }^{2, *}$, Klaus Bogenberger ${ }^{2}$ and Fritz Busch ${ }^{2}$
}

\begin{abstract}
Electrifying mobility-on-demand (MoD) fleets is an important step towards a more sustainable transportation system. With increasing fleet size, MoD operators will be able to participate in the energy exchange market and will have access to time-varying electricity prices. They can benefit from intelligent scheduling of charging processes considering forecasts of electricity prices and vehicle utilization. Considering a long time horizon of, e.g., a day improves scheduling decisions, but electricity prices change in a short interval of 15 minutes; hence, an optimization-based approach needs to overcome challenges regarding computational time. For this reason, we develop a macroscopic model to study the tradeoffs between electricity, battery wear and level-of-service costs. In scenarios with varying fleet size and different numbers of charging units, we compare the performance of several reactive and scheduling policies in a simulation framework based on a macroscopic model. Overall, the results of the study show that an MoD provider with 2000 vehicles could save several thousands of euros in daily operational costs by changing from a state of charge reactive charging strategy to one adapting to the price fluctuations of the electricity exchange market.
\end{abstract}

\section{INTRODUCTION}

The transport sector accounted for the largest share of total energy consumption in Germany in 2017. Moreover, only around $1.5 \%$ of the total amount spent on transportation was covered by electric drive technologies [1]. In the course of climate change, electric mobility with regenerative energy sources is becoming increasingly important to reach the targeted $\mathrm{CO}_{2}$ neutrality by 2050. The electrification of private vehicles, however, is proceeding slowly, which is reflected in the share of electric vehicles still being below $1 \%$ in Germany [2]. By replacing a large number of (fuelpowered) private vehicles by electric vehicles, mobility-ondemand (MoD) systems have the potential to significantly improve the $\mathrm{CO}_{2}$ footprint.

MoD operators using electric fleets can increase their efficiency and profitability by intelligent charging infrastructure [3] and charging strategies. Such strategies determine the combination of charging processes with existing vehicle routing problems [4]. Most studies investigating free-floating MoD systems in urban networks examine reactive charging strategies, i.e. vehicles are sent charging whenever they fall below a certain threshold [5], [6]. Additional to charging lowenergy vehicles, Loeb et al. [7] add a rule to send vehicles charging after being idle for long time and use different charging powers according to the vehicle's state of charge

\footnotetext{
${ }^{1}$ Department of Traffic Engineering at Bundeswehr University Munich, 85577 Neubiberg, Germany

${ }^{2}$ Chair of Traffic Engineering and Control at Technical University $\mathrm{Mu}-$ nich, 80333 Munich, Germany

*Corresponding author: fabian.fehn@tum.de
}

(SOC). Another approach is to extend the routing network by edges that represent charging processes. Therefore, the insertion of charging processes have a planning horizon of typical route lengths. While this horizon might be up to several hours in intercity routes [8], urban context prohibits long time horizons in large-scale problems [9], [10]. Considering the impact of MoD charging processes on the electric overloads and voltage drops provides additional complexity and optimization potential in case the power distribution network and MoD operator are the same entity [11]. Since demand and supply interaction determine the fluctuation of electricity prices, even MoD charging strategies only considering the electricity price might be useful for both operators. In order to increase the ratio of charging in times of low electricity prices, Fehn et al. [12] introduced a rulebased charging strategy taking into account the state of charge of vehicles and dynamic electricity prices. Zhang et al. [13] proposed a smart charging framework to identify the benefits of charging management. Their results suggest that in absence of an electricity price signal charging is likely to take place during the demand peak in the evening.

All before-mentioned urban charging strategies are either reactive or have a short predictive time horizon and charge vehicles at low SOC; scheduling charging processes of vehicles even at a higher SOC before peak hours might decrease the number of vehicles with insufficient SOC in peak hours, thereby increasing the level-of-service in these critical hours. Demand forecasting not only allows spatially re-positioning of vehicles [14], but also estimating future vehicle utilization [15]; hence, demand forecasts enable beneficial scheduling of the number of charging vehicles considering likely vehicle utilization and energy consumption for a long time horizon. Furthermore, this scheduling process can consider the inherent trade-off determining the power of charging processes: fast charging improves the number of available vehicles and therefore level-of-service, but reduces the lifetime of batteries.

The contributions of this study are (i) the development of an MoD charging schedule model considering trade-offs from costs related to charging costs for time-dependent electricity prices, level-of-service and battery wear, (ii) the development of rule- and optimization-based charging scheduling strategies and (iii) the comparison of these strategies with reactive strategies in a case study resembling an MoD system in Munich. 
TABLE I

\section{NOMENCLATURE}

Notation $\mid$ Description

Exogenous Model Parameters

$A_{x}, A_{y} \quad$ edge lengths of rectangular operating area

$v_{i} \quad$ average network velocity during $t_{i}$

$\lambda_{i} \quad$ trip rate during $t_{i}$

$d_{i} \quad$ average trip length during $t_{i}$

$\xi \quad$ penalty for not served customer

$E_{i} \quad$ electricity price during $t_{i}$

$B \quad$ battery size of vehicles

$e^{d} \quad$ distance-dependent energy consumption

$r \quad$ minimal range of battery for available vehicle

$c^{d} \quad$ average distance-dependent wear costs

$N^{V} \quad$ number of fleet vehicles

$N^{C} \quad$ number of charging units

\begin{tabular}{c|c} 
Endogenous Model Variables \\
$S_{i}$ & average state of charge at the beginning of $t_{i}$
\end{tabular}

$C_{i} \quad$ total electricity costs during $t_{i}$

$L_{i} \quad$ level-of-service loss during $t_{i}$

$W_{i} \quad$ power-dependent battery wear costs during $t_{i}$

$T_{i} \quad$ state of charge evolution model during $t_{i}$

$f^{p u} \quad$ distance of pick-up trips

$N_{i}^{t} \quad$ number of concurrent travelers during $t_{i}$

$N_{i}^{a v} \quad$ number of available vehicles during $t_{i}$

$N_{i}^{s} \quad$ number of served customers during $t_{i}$

Decision Variables

\begin{tabular}{l|l}
$p_{i}$ & power of charging processes during $t_{i}$
\end{tabular}

$n_{i} \quad$ number of charging vehicles during $t_{i}$

\section{MODEl DesCription}

The idea of pre-day scheduling charging processes requires forecasts of customer demand and electricity prices. Furthermore, predictive optimization-based charging strategies need to consider many time steps in the future. While the operation of MoD fleets is often modeled using agentbased simulation, the goal of the framework introduced in the subsequent sections is a very fast computational execution. This allows an operator to predict future system behavior over the long time horizon of an entire day. In the following, we will use the notation of Table I.

\section{A. Problem Formulation}

An MoD operator offers a service with electric vehicles in an operating area, which is assumed to be rectangular with edge lengths $A_{x}$ and $A_{y}$ and contains $N^{C}$ charging units. For simplicity, this study focuses on a homogeneous fleet of $N^{V}$ vehicles with battery size $B$ and a distance-dependent energy consumption $e^{d}$. For this study, a day is split into $i \in\{0, \ldots, 95\}$ time periods of length $T=15 \mathrm{~min}$ as this reflects the dynamism of electricity price changes [16]. The operator can charge $n_{i} \leq \min \left(N^{V}, N^{C}\right)$ vehicles with varying power $p_{i}$ in each period, which generates electricity costs and increases the fleet's state of charge in the next time step $S_{i+1}$. The power of charging processes affects the lifetime of batteries; hence, wear costs $W_{i}\left(n_{i}, p_{i}\right)$ are a consequence of the operator's decisions. Moreover, the levelof-service depends on the number of vehicles available to transport passengers. This number is predominantly relying on the fleet size, but also depending on the number of charging vehicles and vehicles with low state of charge. The time-dependent network velocity $v_{i}$, the trip rate $\lambda_{i}$ and the

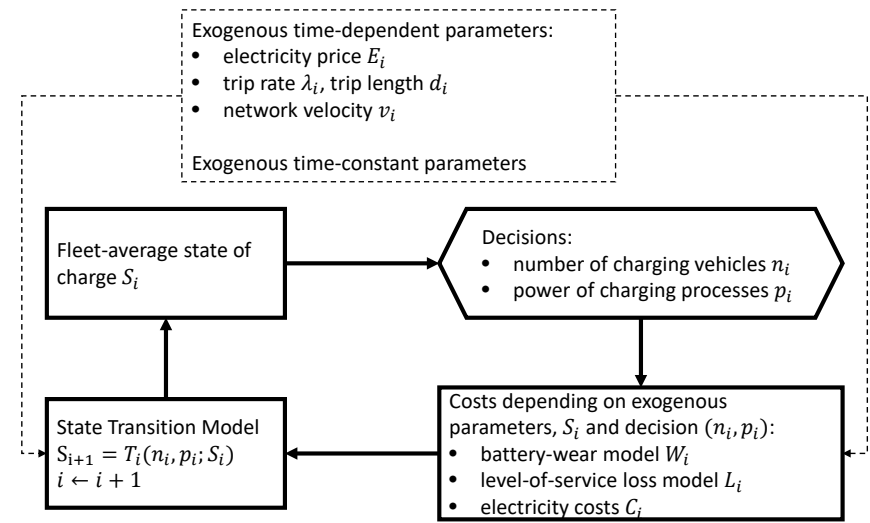

Fig. 1. Flowchart describing the problem formulation: in each time period, the operator makes decisions about the number of charging vehicles and the respective power transfer; the costs and the system state in the next period depend on these decisions and the exogenous variables.

average trip length $d_{i}$ are further impact factors for the levelof-service.

The objective of the operator is to replenish the state of charge of his fleet $\left(S_{95} \geq S_{0}\right)$ in a cost-optimal way. Costs consist of electricity costs $C_{i}\left(n_{i}, p_{i}\right)$, level-of-service losses $L_{i}\left(n_{i}, p_{i} ; S_{i}\right)$ and battery wear $W_{i}\left(n_{i}, p_{i}\right)$. The state of charge evolves according to a state-transition model $T_{i}\left(n_{i}, p_{i} ; S_{i}\right)$.

An operator wants to develop a policy $\pi$ that determines actions $\left(n_{i}, p_{i}\right)$ based on the cost and transition models, the endogenous variable $S_{i}$ and all exogenous variables. The problem with objective function $J$ can be described by

$$
\begin{aligned}
J[\pi] & =\sum_{i=0}^{95} J_{i}\left(n_{i}, p_{i} ; S_{i}\right) \\
& =\sum_{i=0}^{95} C_{i}+L_{i}+W i \\
S_{i+1} & =S_{i}+T_{i}\left(n_{i}, p_{i} ; S_{i}\right) \\
S_{96} & \geq S_{0} \\
0 & \leq S_{i} \leq 1 \\
0 & \leq n_{i} \leq \min \left(N^{V}, N^{C}\right) \\
p_{i} & \in P\left(S_{i}\right)
\end{aligned}
$$

where the dependency on exogenous variables is suppressed for clarity. The flowchart in Fig. 1 summarizes the system dynamics and the sub-models, which will be described in the following sections.

\section{B. Decision Variable Constraints}

There are a few restrictions limiting the decisions $\left(n_{i}, p_{i}\right)$ of an operator. Obviously, the number of concurrently charging vehicles is constrained by both the number of vehicles and the number of charging units. The restrictions on the state of charge $(4,5)$ constrain the allowed solutions for $\left(n_{i}, p_{i}\right)$. While the state-transition model elaborates on the constraint $S_{i} \in[0,1]$, the constraint (4) requires foresight and is therefore treated implicitly by the charging strategies. 
In order to keep the solution space tractable, we limit the power $p_{i}$ of charging vehicles in each time interval to well established power levels derived from Electric Vehicle Database [17]. The values correspond to a 3-phase 16A (11 kW), a CCS $50 \mathrm{~kW} \mathrm{DC} \mathrm{and} \mathrm{CCS} 175 \mathrm{~kW} \mathrm{DC} \mathrm{(48} \mathrm{kW}$ and $124 \mathrm{~kW})$ and an ultra-fast $(163 \mathrm{~kW})$ charging point. Additionally, the rate, at which, energy is transferred to batteries is usually depending on the state of charge; this is modeled by removing the highest power options for a high SOC of the vehicles:

$$
P\left(S_{i}\right)=\left\{\begin{array}{cc}
\{11,48,124,163\} \mathrm{kW} & S_{i} \leq 0.5 \\
\{11,48,124\} \mathrm{kW} & S_{i} \leq 0.7 \\
\{11,48\} \mathrm{kW} & S_{i}>0.7
\end{array}\right.
$$

The threshold values are based on the FASTNED data base [18].

\section{Battery-Wear Model}

The deterioration of batteries consists of two main effects. First, the capacity of batteries decreases gradually, leading to a decrease in range of the vehicle. Second, the batteries' internal resistance increases, resulting in a loss of performance, for example, when accelerating the vehicle. Data collected for Plug In America's (PIA) Tesla Roadster battery study suggests that, on average, a battery pack will have between 80 and $85 \%$ of original capacity after 100000 miles [19].

According to the German test lab of SGS Group, battery wear strongly depends on charging behavior. In particular, full charging cycles, in which batteries reach their maximum depth of discharge, have a direct negative effect on the lifetime of the battery [20]. Intelligent battery management systems already consider this effect and prevent the battery from being deeply discharged or overcharged. This study prevents deeply discharging of batteries by not assigning vehicles with low SOC to serve customers. In addition, the charging power also plays an important role. During socalled fast charging, local overheating can occur, which leads to increased deterioration. For a detailed literature review on the physical phenomena and degradation mechanisms during fast charging, we refer to the literature review of Tomaszewska et al. [21].

For this study, the battery wear model accounts for general gradual degradation $\left(w_{0}\right)$ and an additional power-dependent component $\left(w_{1}\right)$.

$$
W_{i}\left(n_{i}, p_{i}\right)=\left(w_{0}+w_{1} \cdot p_{i}\right) \frac{n_{i} \cdot p_{i} \cdot T}{B}
$$

\section{Level-of-Service Model}

The level-of-service is often derived from very detailed agent-based simulation models. Since a predictive algorithm requires quick simulation of many future steps, possibly even for multiple choices of $\left(n_{i}, p_{i}\right)$, we develop a novel macroscopic model that approximates $L_{i}$ and its dependencies.

The amount of time vehicles need to drive customers from their origin to their destination is given by $\lambda_{i} \cdot T \cdot d_{i} / v_{i}$. A division of this quantity by $T$ returns the average number of concurrently traveling customers $N_{i}^{t}$.

$$
N_{i}^{t}=\left\lfloor\frac{\lambda_{i} \cdot d_{i}}{v_{i}}\right\rfloor
$$

$\lfloor x\rfloor$ rounds $x$ down to the nearest integer value. The operator can use available vehicles to serve this demand. Vehicles are not considered available if they are charging or if their remaining range is below $r$, i.e. a state of charge smaller than $r \cdot e^{d} / B$. The number of charging vehicles is $n_{i}$ and the number of vehicles with insufficient range is estimated via $S_{i}$ by assuming a certain distribution of vehicle battery levels, which has a domain of $[0,1], \mu_{i}=S_{i}$ and a variance of 0 for $S_{i} \in\{0,1\}$. We assume that the operator utilizes vehicles similarly and that the vehicles' state of charge spreads around the fleet average by choosing a truncated normal distribution $D^{T N}$ of vehicle battery levels with $\mu_{i}=S_{i}$ and $\sigma_{i}=1-$ $4 *\left(S_{i}-0.5\right)^{2}$ truncated to the domain $[0,1]$.

Let $s$ be a random variable drawn from this distribution denoting the state of charge of a single vehicle. Consequently, the number of available vehicles $N_{i}^{a v}\left(n_{i} ; S_{i}\right)$ can be estimated from the probability that a vehicle has sufficient range and is not charging.

$$
N_{i}^{a v}=\left\lfloor N^{v} \cdot P\left[s \geq \frac{r \cdot e^{d}}{B} \mid s \sim D_{[0,1]}^{T N}\left(\mu_{i}, \sigma_{i}\right)\right]-n_{i}\right\rfloor
$$

Since only available vehicles can meet the passenger demand, we define the number of served customers $N_{i}^{s}=$ $\min \left(N_{i}^{t}, N_{i}^{a v}\right)$. The level-of-service loss $L_{i}$ consists of (i) a penalty for customers that cannot be served and (ii) distancedependent wear costs for $f^{p u}$ (the empty mileage of pick-up trips), which depends on the number of concurrent travelers and available vehicles per area.

$$
L_{i}=L_{i}^{n s}+L_{i}^{p u}=\xi \cdot\left(N_{i}^{t}-N_{i}^{s}\right)+c^{d} \cdot f^{p u}\left(N_{i}^{s}, N_{i}^{a v}\right)
$$

Note that $c^{d}$ only contains the wear cost component and excludes battery wear and energy cost components. Costs related to a lower state of charge from additional vehicle mileage are captured by the electricity and battery wear costs at a later point in time due to the stability condition $S_{96} \geq S_{0}$. We develop a simulation model to evaluate the average distance of empty pick-up trips $f^{p u}$ only based on the macroscopic quantities $\left(N_{i}^{s}, N_{i}^{a v} ; A_{x}, A_{y}\right)$. The model is using Monte-Carlo experiments and is based on sequentially matching a traveler with the remaining number of available vehicles. The model is explained and $f^{p u}$ is illustrated in Appendix A.

\section{E. State-Transition Model}

The evolution of the state of charge from $S_{i}$ to $S_{i+1}$ is determined by the transmitted energy at charging stations and reductions due to vehicles traveling. The transferred energy during time period $t_{i}$ is given by $n_{i} \cdot p_{i} \cdot T$. On average, $N_{i}^{S}$ vehicles drives $d_{i}$ kilometers to transport customers from their origin to their destination. Additionally, the fleet needs to drive $f^{p u}$ kilometers to pick-up customers.

$$
\bar{T}_{i}=\frac{n_{i} \cdot p_{i} \cdot T-e^{d}\left(N_{i}^{s} \cdot d_{i}+f^{p u}\left(N_{i}^{s}, N_{i}^{a v}\right)\right)}{N^{V} \cdot B}
$$


Equations (10) and (11) for $N_{i}^{s}$ and $N_{i}^{a v}$ show that the state evolution is actually depending on all exogenous input parameters.

Note that for small values of $S_{i}$, the number of available vehicles $N_{i}^{a v}$ and hence the mileage and reduction in state of charge are very small. Nevertheless, this model does not guarantee the hard constraint $S_{i} \geq 0$ of equation (5). For simplicity, $S_{i+1}$ will be set to 0 in the unlikely event that $S_{i}+\bar{T}_{i}<0$, because these solutions are anyway far from optimal due to very high losses in the level-of-service. Similarly, $S_{i+1}$ is set to 1 in case $S_{i}+\bar{T}_{i}>1$. Solutions with decisions leading to this case are anyway non-optimal since the electricity costs $C_{i}$ grow without any benefit. Hence, relevant charging strategies do not violate these two constraints in practice. Altogether, the state transition function reads

$$
T_{i}=\min \left(\max \left(\overline{T_{i}}, 1-S_{i}\right),-S_{i}\right)
$$

with $\bar{T}_{i}$ determined by equation (13).

\section{Charging Strategies}

\section{A. Short-Term Charging Strategy}

In reactive charging strategies, the $\mathrm{MoD}$ operator sends a vehicle to recharge in case of low state of charge. These kind of policies can be represented in the introduced model by setting the targeted number $n_{i}^{\text {target }}$ of vehicles to be charged during interval $t_{i}$ to the number of vehicles with state of charge below the threshold $s_{\text {lim }}$. Obviously, the actual number of charging vehicles is also constrained by the number of charging units.

$$
\begin{aligned}
n_{i}^{\text {target }} & =\left\lfloor N^{v} \cdot P\left[s \leq \frac{r \cdot e^{d}}{B} \mid s \sim D_{[0,1]}^{T N}\left(\mu_{i}, \sigma_{i}\right)\right]\right\rfloor \\
n_{i} & =\min \left(n_{i}^{\text {target }}, N^{C}\right)
\end{aligned}
$$

There are several possibilities to set the charging power. Two possible strategies are described in the following and tested in the case study.

1) State of Charge Dependent Short-Term Charging Strategy (SOC-ST): The first policy aims to keep the SOC at a certain level at all times. Hence, the operator selects the power $p_{i}$ of each interval according to $S_{i}$ :

$$
p_{i}=\left\{\begin{array}{cc}
163 \mathrm{~kW} & S_{i} \leq 0.5 \\
124 \mathrm{~kW} & S_{i} \leq 0.7 \\
48 \mathrm{~kW} & S_{i} \leq 0.8 \\
11 \mathrm{~kW} & S_{i}>0.8
\end{array}\right.
$$

2) Electricity-Price Dependent Short-Term Charging Strategy $(E P-S T)$ : In this policy, the operator sets the power of charging processes to the minimal value if the electricity price is above the average electricity price $\bar{E}$ and to the maximal power value in the other case.

$$
p_{i}= \begin{cases}\max \left(P\left(S_{i}\right)\right. & E_{i} \leq \bar{E} \\ \min \left(P\left(S_{i}\right)\right. & E_{i}>\bar{E}\end{cases}
$$

In order to reach the initial state of charge at the end of the day, both short-term strategies apply the following policy after 22:00 in case $S_{i}<S_{0}$ :

$$
\begin{aligned}
p_{i} & =\max \left(P\left(S_{i}\right)\right) \\
n_{i} & =\min \left(1+\left\lfloor\frac{N^{V} \cdot B}{p_{i} \cdot T}\left(S_{0}-S_{i}\right)\right\rfloor, N^{C}\right)
\end{aligned}
$$

While this does not guarantee condition (4), simulations show the desired results in the case study.

\section{B. Overnight Charging Strategy (OVNC)}

This strategy corresponds to a naive approach for typical demand and electricity price curves, which often show that the period between 20:00 and 05:00 might be a good time to charge the fleet. At time step $i$, the policy aims to have fully charged vehicles at 05:00 and the initial SOC $S_{0}$ at 24:00. First, the targeted energy difference $\Delta S^{\text {target }} \in\left\{1.0, S_{0}\right\}$ and the next target time period $i^{\text {target }} \in\{20,96\}$ are set. Then the power $p_{i}$ is determined by assuming $N^{C}$ vehicles can charge during the time periods $j$ until $i^{\text {target }}$. For the morning (night) hours, the power $p_{i} \in P\left(S_{i}\right)$ is determined by the largest (smallest) value that is below (above) the target power $p^{\text {target }}$ with

$$
\sum_{j=i}^{i^{\text {target }}} N^{C} \cdot p^{\text {target }} \cdot T=N^{V} \cdot B \cdot \Delta S^{\text {target }}
$$

Next, the number of charging vehicles $n_{i}$ is either 0 for $(20 \leq i<80)$ or computed from

$$
n_{i}=\frac{N^{V} \cdot B \cdot \Delta S^{\text {target }}}{p_{i} \cdot T\left(i^{\text {target }}-i\right)}
$$

\section{Pre-Day Scheduling of Charging Processes}

The optimization problem $\min _{\pi} J[\pi]$ considering the model constraints represents a scheduling problem. Due to the large amount of time steps and considerable number of $\left(n_{i}, p_{i}\right)$ options per time step, the solution space of order $O\left(m^{96}\right)$ (with $m=N^{C} \cdot|P(S)|$ ) prohibits the application of exact dynamic programming. Therefore, we develop a rulebased approach and subsequently use it for an approximate dynamic programming strategy.

1) Rule-Based Pre-Day Scheduling (RB-PDS): The key idea of the rule-based approach is to use approximations of the objective function and the state-transition model by (i) prioritizing to serve demand, (ii) assuming that all demand can be met and (iii) neglecting the state of charge dependence of $f^{p u}$. The latter is achieved by assuming a constant rate of $25 \%$ empty mileage, i.e. $f^{p u, R B} \approx 0.25 \cdot N_{i}^{t} \cdot d_{i}$. These simplifications result in $J_{i}^{R B}=J_{i}\left(n_{i}, p_{i}\right)$ and $T_{i}^{R B}=$ $T_{i}\left(n_{i}, p_{i}\right)$ becoming independent of $S_{i}$. As a consequence, the state of charge $S_{i}^{R B}$ becomes only dependent on the energy of charging processes prior to time period $t_{i}$, i.e. $S_{i}^{R B}=f\left(\sum_{j=0}^{i-1} n_{j} \cdot p_{j}\right)$.

These approximations enable a rule-based approach, which has following priorities: 1) serving demand, 2) sustainability (i.e. low battery wear) and 3) adaption to electricity prices. The algorithm performs the following steps:

- compute number of vehicles that are required to serve the demand $N_{i}^{t}$ and possibly idle vehicles $N^{V}-$ 
$N_{i}^{t}$ for all time periods $i$, the maximal number of charging vehicles per period are given by $N_{i}^{R B}=$ $\min \left(N^{C}, \max \left(N^{V}-N_{i}^{t}, 0\right)\right)$

- compute initial state of charge evolution without charging processes assuming all customers can be served:

$$
S_{i}^{R B}=S_{0}-1.25 \cdot e^{d} \sum_{j=0}^{i-1} N_{j}^{t} \cdot d_{j}
$$

- determine constant charging power $p \in P(S)$ by finding the lowest value of $p$ with

$$
\sum_{i=0}^{95} N_{i}^{R B} \cdot p \cdot T \geq N^{V} \cdot B \cdot\left(S_{0}-S_{96}^{R B}\right)
$$

- add charging processes $n_{i}$ to time periods $t_{i}$ in order to guarantee $S_{i} \geq 0 \forall i$ and then $S_{96}^{\text {lin }} \geq S_{0}$. In case $S_{j}^{R B}<0$ for any $t_{j}$ (starting from $j=0$ ), the following loop terminates when $S_{j}^{R B} \geq 0$ until $S_{i}^{R B} \geq 0 \forall i \in$ $\{0 . .96\}$. After that, the loop continues with $j=96$ while $S_{96}^{R B}<S_{0}$.

- find the time period $i *<j$ with the lowest energy price and set $n_{i *}$ to $N_{i}^{R B}$

- remove time period $i *$ from consideration for future loop iterations

- update $\left\{S_{i}^{R B}\right\}_{i=1 . .96}$ :

$$
S_{i}^{R B} \leftarrow\left\{\begin{array}{cl}
S_{i}^{R B} & i \leq i * \\
S_{i}^{R B}+n_{i-1} \cdot p \cdot T & i>i *
\end{array}\right.
$$

2) Pre-Day Scheduling by Approximate Dynamic Programming with Policy-Application (PA-PDS): The (RBPDS) policy approximates the objective and state-transition functions to achieve non-myopic decisions. However, the rules limit the decision space actually being reached in each step; for example, $p_{i}$ stays the same throughout the day. A more advanced approximate dynamic programming approach allows spanning the complete decision space for the current step while estimating the impact of this decision on the rest of the time horizon by applying a policy. Hence, the complexity of this approach is $96 \cdot O(m \cdot c)$, where $c$ is the complexity of one step of the applied policy, and therefore, computationally feasible (if the underlying policy is). Mathematically speaking, a policy-application strategy with underlying policy $\pi$ assigns the action $\left(n_{i}^{*}, p_{i}^{*}\right)$ for each interval $i$ that is determined by

$$
\arg \min _{n_{i}, p_{i}}\left(J_{i}\left(n_{i}, p_{i} ; S_{i}\right)+\left[\sum_{j=i+1}^{95} J_{j}\left(n_{j}^{\pi}, p_{j}^{\pi} ; S_{j}^{\pi}\right)\right]\right)
$$

and the system dynamics and constraints in equations (37), where $\left(n_{j}^{\pi}, p_{j}^{\pi}\right)$ are the actions taken according to policy $\pi$ at time step $j$ with state of charge $S_{j}$. In order to save computation time, we vary $n_{i}$ in steps of 5 in case there are at least 25 available options for $n_{i}$ and in steps of 10 in case there are at least 100 options. Test simulations with $n_{i}$ steps of 5 for more than 100 options only resulted in slightly better objective function values $(\leq 0.1 \%)$ while being computationally far more expensive.

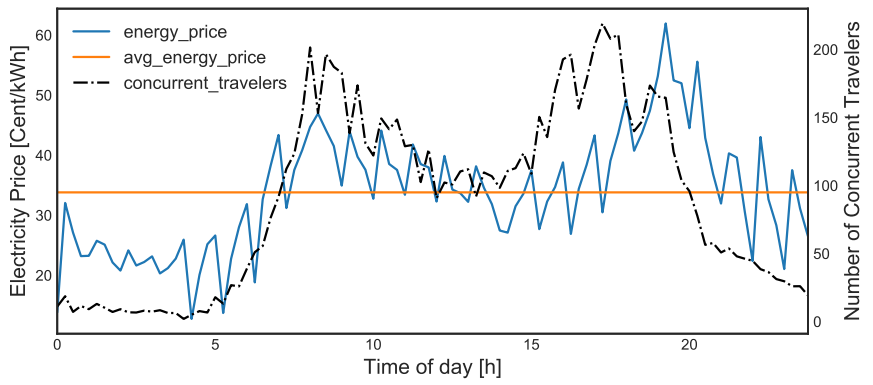

Fig. 2. Evolution of scenario-average energy price and number of concurrently travelers over time.

In this study, we apply the (RB-PDS) policy of the previous section at each time step $i$, with the modification that the planning horizon of the algorithm starts at $i$ instead of 0 .

\section{CASE Study}

\section{A. Scenario Setup}

Simulations are defined by a certain set of the exogenous parameters named in Table I. This case study at hand is based on data representing the city of Munich. The operating area ( $A_{x}=7 \mathrm{~km}$ and $A_{y}=10 \mathrm{~km}$ ), the trip rates $\lambda_{i}$ and trip lengths $d_{i}$ are based on a prior MoD study [22]. The average velocities per time interval are extracted from a traffic microsimulation model [23]. The penalty factor for demand not served is chosen to be $5 €$ per customer.

For this study, energy prices $E_{i}$ were downloaded from the European Energy Exchange Market (EPEX SPOT SE) for a period of 14 days between Monday 2019-10-07 00:00 and Sunday 2019-10-20 24:00 [16]. The battery size $B=$ $42 \mathrm{kWh}$ and energy consumption $e^{d}=0.131 \mathrm{~kW} / \mathrm{km}$ represent vehicle data from EV Database [17]. The battery wear cost parameters $w_{0}$ and $w_{1}$ are estimated from the manufacturer stating replacement costs between 5,500 $€$ and $7,700 €$ after 500 to 1500 charging cycles. We assumed costs of $6,750 €$ spread on 1500 or 500 cycles if vehicles are charged exclusively with 11 or $164 \mathrm{~kW}$, respectively. Furthermore, we assume that the minimal range parameter $r=31.2 \mathrm{~km}$, which assigns vehicles being considered available to serve customer demand, corresponds to $10 \%$ of the battery size. This value seems reasonable since it prohibits higher battery degradation at very low SOCs and ensures users' confidence that they will reach their destination. The distance-dependent cost parameter $c^{d}$, which does not include energy and battery wear costs, is estimated from the full cost estimates by ADAC [24].

Fig. 2 illustrates the time evolution of concurrent travelers and the energy price (average of simulated days). An operator will choose the fleet size according to the number of concurrent travelers. The base scenario of this study employs a fleet of $N^{V}=2000$ electric vehicles. Furthermore, we assume a charging infrastructure with $N^{C}=200$ charging units and an initial SOC $S_{0}=70 \%$ at 00:00. 

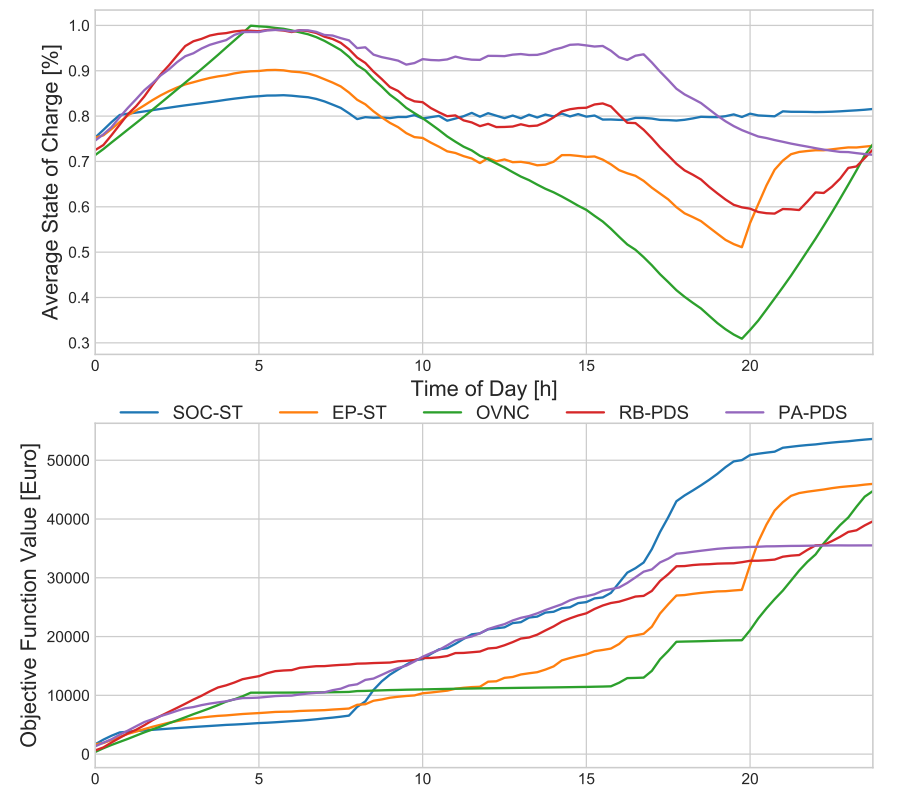

Fig. 3. Evolution of the objective function (top) and the fleet-average state of charge (bottom) over time for the different charging strategies in the base scenario.

We study the variation of the initial SOC $S_{0} \in$ $\{0.8,0.7,0.6,0.5\}$, the fleet size $N^{V} \in\{2000,2500\}$ and the number of charging units $N^{C} \in\{100,200,300\}$.

\section{B. Results}

Fig. 3 shows the time evolution of the fleet-average SOC and objective function value, where each line represents the average of the 14 simulated days. The SOC-ST strategy aims to keep the fleet-average SOC at a high level throughout the day. Consequently, vehicles mostly charge during and shortly after the demand peaks leading to regions with stark slope in the objective function. Since the energy price is rather high (more than $0.4 € / \mathrm{kWh}$ on average), this policy generates the highest charging costs $(28 \mathrm{k} €)$. Only the to short-term policies (SOC-ST, EP-ST) generate level-of-service costs of more than $10 \mathrm{k} €$ because vehicles are charging during peak hours. The OVNC policy produces the highest battery wear costs as this policy requires higher charging power to transfer a similar amount of energy in a restricted time period. The level-of-service is sufficient for most of the time during the day. Hence, the objective function is almost constant between 05:00 and 16:00. The rather low value of 50\% SOC during the evening demand peak causes a larger number of not available vehicles compared to the other policies. Therefore, the level-of-service costs are rather high with approximately $9 \mathrm{k} €$. Since the times of low energy prices often coincide with the OVNC charging time period, the total charging costs are similar in the EP-ST and the OVNC policies. Similar to the OVNC policy, the pre-day scheduling approaches (RBPDS, PA-PDS) use the morning hours to charge all vehicles as full as possible. The RB-PDS policy ignores state of charge dependent cost contributions in $f^{p u}$ and hence puts less weight on the SOC of the fleet than the better PA-PDS
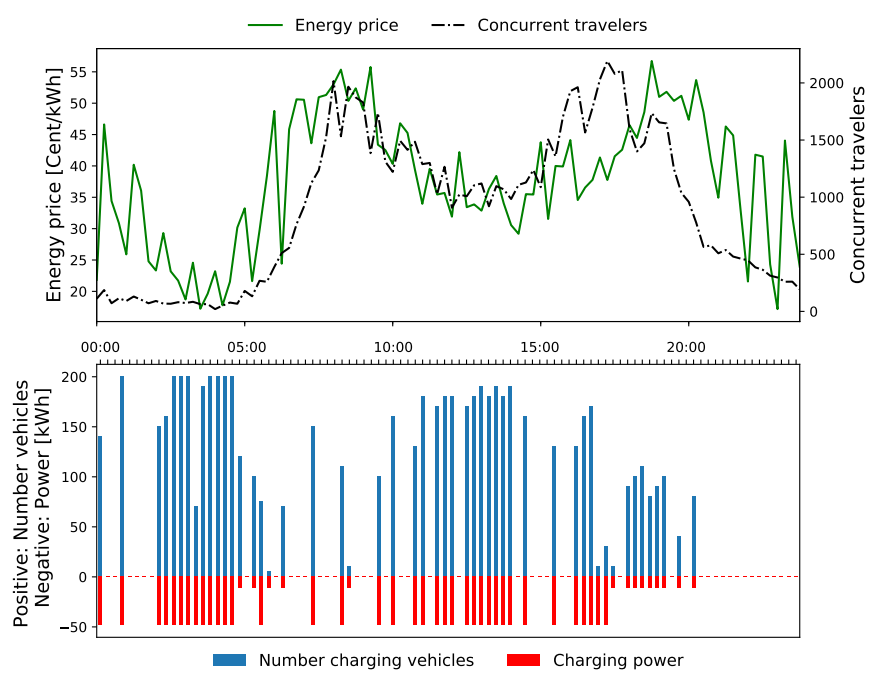

Fig. 4. Time evolution for a single day (Wednesday, 2019-10-16) of the base scenario showing the energy price and the number of concurrent travelers (top), and the number of charging vehicles and the charging power according to the PA-PDS policy (bottom).

policy. The scheduling approaches generate solutions which save more than $5000 €($ RB-PDS) and more than $9000 €$ per day (PA-PDS) in operational costs, compared to the other charging strategies.

Even though the PA-PDS policy already outperforms the other policies, we still recognize further potential for improvement. The non-optimal decisions are caused by the bias inherently generated in the approximation of $\min \left(\sum_{j=i+1}^{95} J_{j}\right)$ by a policy iteration approach. Fig. 4 shows that the policy tries to avoid charging with lower power in times of high energy prices, but it might be that delaying some charging processes until after 22:00 might lead to an even better solution.

Fig. 5 illustrates results of the scenarios with $S_{0}, N^{V}$ and $N^{C}$ variation. The initial SOC $S_{0}$ is an input parameter to the simulations, but can also be viewed as a policy parameter. It determines the ratio of charging processes in the morning hours over the night hours. It is noticeable that the PA-PDS is capable of similar performance metrics in the chosen range of $S_{0}$. The SOC-ST strategy performs worse with lower $S_{0}$ since the battery wear is high from initial fast charging processes to increase the SOC to $80 \%$ as soon as possible; for $S_{0}<0.8$ there are additional costs due to a total energy surplus $\left(S_{96}>S_{0}\right)$. The overnight policy profits from lower $S_{0}$ because less power is required to replenish the SOC after 22:00. As expected, scenarios with a higher number of vehicles $N^{V}$ produce less operational costs. The levelof-service component is naturally better and the operator can delay charging processes without large level-of-service losses. Furthermore, the simulations show that an increase in the number of charging units from 200 to 300 hardly brings any benefits, while there are noticeable differences between 100 and 200. In general, the importance or difference in performance of the various policies decreases with larger fleet 

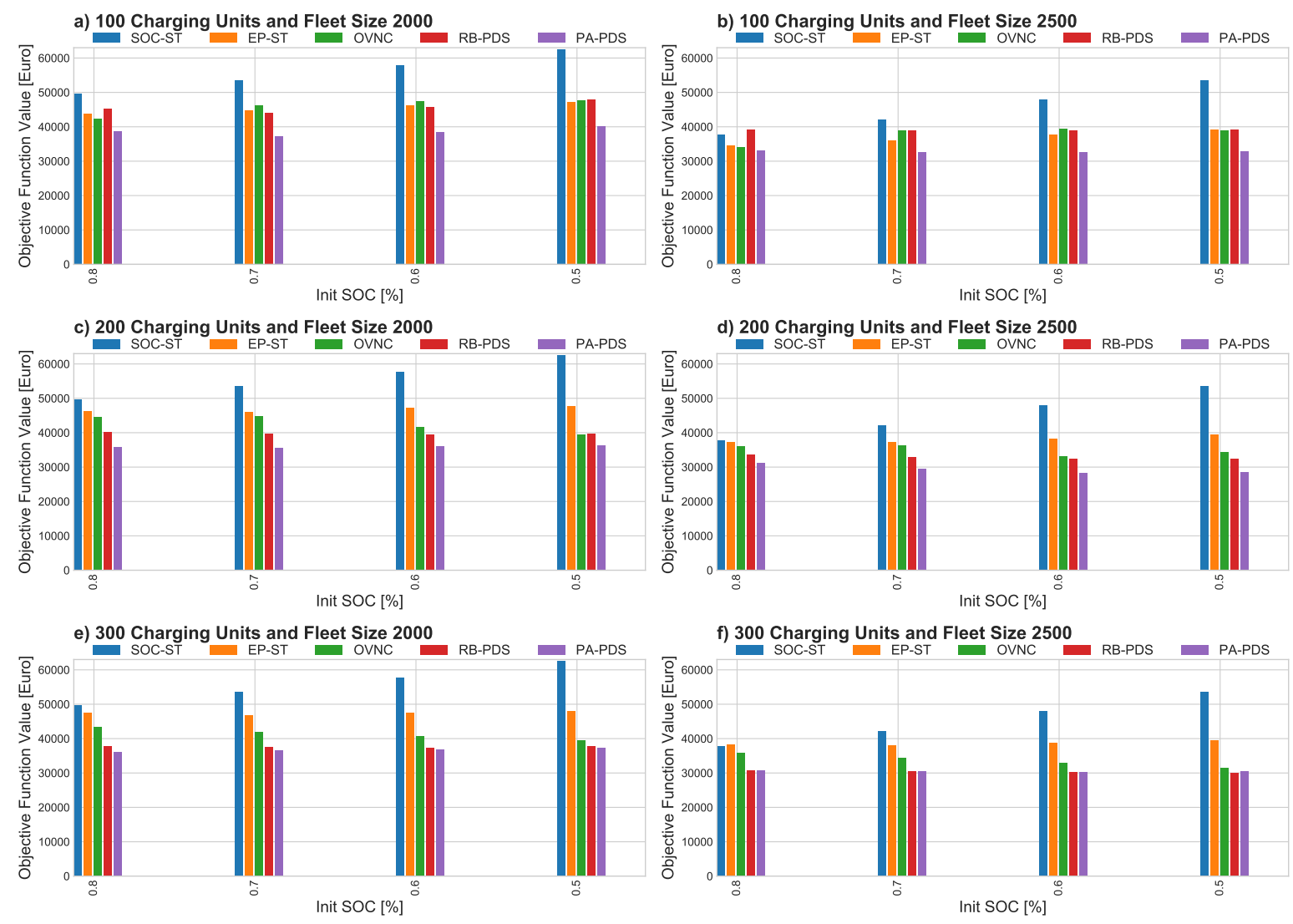

Fig. 5. Comparison of objective function results for different scenarios and policies averaged over the simulated days.

sizes. Besides the shown daily operational costs, operators have to consider fix costs (investment, maintenance, tax, etc.) in their decision of $N^{V}$ and $N^{C}$.

\section{CONCLUSION}

Larger electrified MoD fleets will have the opportunity to participate in the energy exchange market. This market is characterized by time-dependent electricity prices changing every 15 minutes. A very fast simulation model and an approximate dynamic programming policy were developed to find a near-optimal scheduling policy, which balances tradeoffs between electricity, battery wear and level-of-service costs for a time horizon of one day. We compared the performance of this scheduling strategy with several policies in various scenarios in a simulation framework based on a macroscopic model. Results show that an operator with 2000 vehicles can save a considerable amount of several thousands of euros per day when changing from a reactive charging strategy, which only recharges vehicles with low SOC, to a scheduling strategy. The low computational effort of the simulation model is also convenient for an optimization of the number and type of vehicles, i.e. battery size and energy consumption, and the number and type of charging units.

The validity of these results depends on the validity of the macroscopic model. Especially, the battery wear model, the vehicle SOC distribution and the level-of-service model contain many simplifications. The approximations of these models need to be verified by data or more detailed models. Furthermore, real MoD operators require a charging control strategy that assigns vehicles to charging stations. For that purpose, we intend to integrate the planning decisions of the scheduling policies within an agent-based real-time simulation [25]. Such a framework allows the inclusion of real electricity price forecasts and the evaluation of impacts from forecast errors. Finally, building a level-of-service and statetransition model considering ride-pooling and the application of different approximate dynamic programming methods represent other very interesting extensions.

\section{APPENDIX}

\section{A. Computing Average Pick-Up Distances in Mobility-On- Demand Systems from Macroscopic Parameters}

The average distance $f^{p u}\left(1, N^{a v}\right)$ between one customer and the next available vehicle is depending on the spatial density of available vehicles. Hence, keeping all other parameters constant, the area parameters $A_{x}, A_{y}$ increase and $N^{a v}$ decrease $f^{p u}$. The number of concurrent customers $N^{t}$ increases $f^{p u}$, because other customers have to be assigned to vehicles, which reduces the effective available vehicle density. In order to quantify these relations, this study assumes uniform distribution of vehicles and customers within the operating area. This limitation can easily be lifted by applying spatially weighted distributions. 


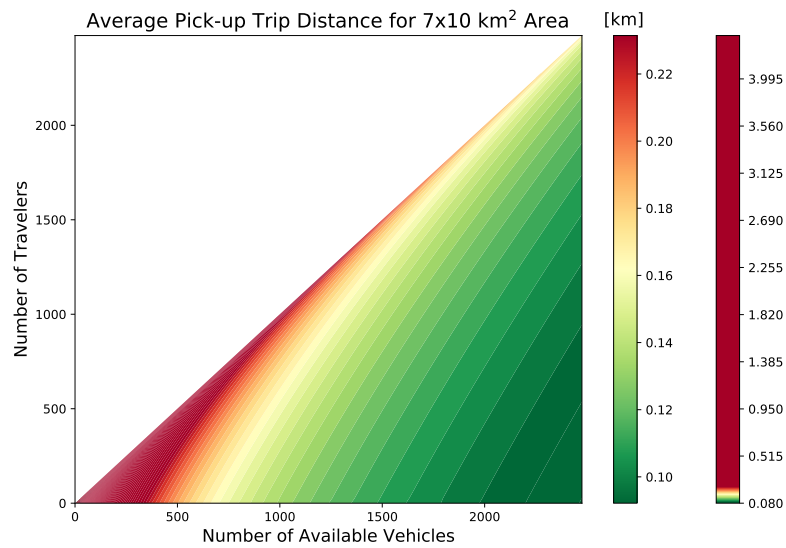

Fig. 6. Contour plot for the average distance of empty pick-up trips $f^{u p}\left(N^{s}, N^{a v} ; A_{x}, A_{y}\right) / N^{t}$ in the assumed operating area.

Currently, $N^{a v}$ vehicle points are sampled from the distribution. Afterwards, the coordinates of a customer point are randomly drawn from within the operating area and the shortest distance of this point to any of the vehicle points is determined. To achieve statistical stability, we iterate this process for 10000 customer points, and repeat it for at least 100 vehicle point distributions and until the relative standard error is below $1 \%$. This procedure returns the final mean value as $f^{p u}\left(1, N^{a v} ; A_{x}, A_{y}\right)$. The computation of the total empty distance of pick-up trips $f^{p u}$ for $N^{s}>1$ can be performed with the approximation of sequential assignments of customers with reduced density of available vehicles:

$$
f^{p u}\left(N^{s}, N^{a v} ; A_{x}, A_{y}\right)=\sum_{i=0}^{N^{s}-1} f^{p u}\left(1, N^{a v}-i\right)
$$

In this study, we do not consider the case $N^{t}>N^{a v}$, because only $N^{a v}$ customers can be served. We assume that the operator does not discriminate customer rejections based on the pick-up distance. The extension of the model for this case would also be straightforward, though. We illustrated $f^{p u}\left(N^{s}, N^{a v}\right)$ for the case study area with edges $A_{x}=7 \mathrm{~km}$ and $A_{y}=10 \mathrm{~km}$ in Fig. 6 .

\section{ACKNOWLEDGMENT}

The authors thank the German Federal Ministry for the Environment, Nature Conservation and Nuclear Safety and the German Federal Ministry for the Economic Affairs and Energy for funding through the projects "City2Share" and "Muenchen elektrisiert".

\section{REFERENCES}

[1] Energieverbrauch nach Energieträgern, Sektoren und Anwendungen. [Online]. Available: https://www.umweltbundesamt.de/daten/energie/

[2] Kraftfahrbundesamt. (2019) Stock of private vehicles according to energy source 2010-2019. [Online]. Available: https://www.kba.de/ DE/Statistik/Fahrzeuge/Bestand/Umwelt/2019_b_umwelt_z.html

[3] F. Dandl, T. Niels, and K. Bogenberger, "Design and control of park $\&$ charge lanes for carsharing services with highly-automated electric vehicles," in 21st IFAC World Congress, 2020.

[4] C. Bongiovanni, M. Kaspi, and N. Geroliminis, "The electric autonomous dial-a-ride problem," Transportation Research Part B: Methodological, vol. 122, pp. 436-456, 2019.
[5] T. D. Chen and K. M. Kockelman, "Operations of a shared, autonomous, electric vehicle fleet: implications of vehicle \& charging infrastructure decisions," Transportation Research Part A: Policy and Practice, vol. 94, pp. 243-254, 2016.

[6] F. Dandl and K. Bogenberger, "Comparing future autonomous electric taxis with an existing free-floating carsharing system," IEEE Transactions on Intelligent Transportation Systems, vol. 20, no. 6, pp. 2037 2047, 2019.

[7] B. Loeb, K. M. Kockelman, and J. Liu, "Shared autonomous electric vehicle (saev) operations across the austin, texas network with charging infrastructure decisions," Transportation Research Part C: Emerging Technologies, vol. 89, pp. 222-233, 2018.

[8] H. Zhang, C. J. R. Sheppard, T. E. Lipman, and S. J. Moura, "Joint fleet sizing and charging system planning for autonomous electric vehicles,' IEEE Transactions on Intelligent Transportation Systems, pp. 1-14, 2019.

[9] L. Li, D. Lin, T. Pantelidis, J. Chow, and S. E. Jabari, "An agent-based simulation for shared automated electric vehicles with vehicle relocation*," in 2019 IEEE Intelligent Transportation Systems Conference (ITSC). IEEE, 2019, pp. 3308-3313.

[10] R. Zhang, F. Rossi, and M. Pavone, "Model predictive control of autonomous mobility-on-demand systems," IEEE International Conference on Robotics and Automation (ICRA), pp. 1382-1389, 2016.

[11] A. Estandia, M. Schiffer, F. Rossi, E. C. Kara, R. Rajagopal, an M. Pavone, "On the interaction between autonomous mobility on demand systems and power distribution networks - an optimal power flow approach," 2019.

[12] F. Fehn, F. Noack, and F. Busch, "Modeling of mobility on-demand fleet operations based on dynamic electricity pricing," in 2019 6th International Conference on Models and Technologies for Intelligent Transportation Systems (MT-ITS). IEEE, 2019, pp. 1-6.

[13] T. Z. Zhang and T. D. Chen, "Smart charging management for shared autonomous electricvehiclefleets: A puget sound case study," Transportation Research Part D, pp. 1-14, 2019.

[14] F. Dandl, M. Hyland, K. Bogenberger, and H. S. Mahmassani, "Evaluating the impact of spatio-temporal demand forecast aggregation on the operational performance of shared autonomous mobility fleets," Transportation, vol. 114, pp. 462-484, 2019.

[15] — - "Dual-horizon forecasts and repositioning strategies for operating shared autonomous mobility fleets," in 99th Annual Meeting of Transportation Research Board, 2020.

[16] E. E. Exchange. (2019) EPEX SPOT SE: Kontinuierlicher IntradayHandel. [Online]. Available: www.epexspot.com

[17] E. V. Database. (2019) Tesla Model 3 Standard Range performance. [Online]. Available: https://ev-database.uk

[18] FASTNED. (2019) 175- / $350 \mathrm{kw}$ fast chargers. [Online]. Available: https://support.fastned.nl/hc/en-gb/articles/115015420127175-350-kW-fast-chargers

[19] T. Saxton. (2013) Plug In America's Tesla Roadster battery study. [Online]. Available: http://pop.h-cdn.co/assets/cm/15/06/ 54d152d48c15c_-_PIA-Roadster-Battery-Study.pdf

[20] H. Detlef. (2015) Alter reduziert Reichweite: Wie lange lebt die Batterie im Elektrofahrzeug? [Online]. Available: https://www.sgsgroup.de/ /media/Local/Germany/Documents/ White\%20Papers/SGS_Batterie_Lebensdauer_im_Elektrofahrzeug_DE_ 1015. ash $x$ ?force $=1$

[21] A. Tomaszewska, Z. Chu, X. Feng, S. O'Kane, X. Liu, J. Chen, C. Ji, E. Endler, R. Li, L. Liu, Y. Li, S. Zheng, S. Vetterlein, J. Gao, M. Du, M. Parkes, M. Ouyang, M. Marinescu, G. Offer, and B. Wu, "Lithiumion battery fast charging: A review," eTransportation, vol. 1, 2019.

[22] R. Engelhardt, F. Dandl, A. Bilali, and K. Bogenberger, "Quantifying the benefits of autonomous on-demand ride-pooling: A simulation study for munich, germany," in 2019 IEEE Intelligent Transportation Systems Conference (ITSC). IEEE, 2019, pp. 2992-2997.

[23] F. Dandl, B. Bracher, and K. Bogenberger, "Microsimulation of an autonomous taxi-system in Munich," in 5th IEEE International Conference on Models and Technologies for Intelligent Transportation Systems (MT-ITS), 2017, pp. 833-838.

[24] ADAC. (2019) ADAC Autokostenrechner. [Online]. Available: https://www.adac.de/infotestrat/autodatenbank/autokosten/

[25] F. Dandl, K. Bogenberger, and H. S. Mahmassani, "Autonomous mobility-on-demand real-time gaming framework," in 2019 6th International Conference on Models and Technologies for Intelligent Transportation Systems (MT-ITS). IEEE, 2019, pp. 1-10. 